\title{
Expression of Plxdc2/TEM7R in the developing nervous system of the mouse
}

\author{
Suzanne F.C. Miller ${ }^{\mathrm{a}}$, Kristen Summerhurst ${ }^{\mathrm{d}}$, Annette E. Rünker ${ }^{\mathrm{a}}$, Géraldine Kerjan ${ }^{\mathrm{b}}$, \\ Roland H. Friedel ${ }^{\mathrm{c}}$, Alain Chédotal ${ }^{\mathrm{b}}$, Paula Murphy ${ }^{\mathrm{d}}$, Kevin J. Mitchell ${ }^{\mathrm{a}, *}$ \\ ${ }^{a}$ Smurfit Institute of Genetics, Trinity College Dublin, Dublin 2, Ireland \\ ${ }^{\mathrm{b}}$ Centre National de la Recherche Scientifique UMR7102, Université de Paris 6, Case 12, 9 Quai Saint-Bernard, 75005 Paris, France \\ ${ }^{\mathrm{c}}$ Department of Biological Sciences, Howard Hughes Medical Institute, Stanford University, Stanford, CA 94305, USA \\ ${ }^{\mathrm{d}}$ Department of Zoology, Trinity College Dublin, Dublin 2, Ireland
}

Received 21 September 2006; received in revised form 14 November 2006; accepted 10 December 2006

\begin{abstract}
Plexin-domain containing $2(P l x d c 2)$ is a relatively uncharacterised transmembrane protein with an area of nidogen homology and a plexin repeat (PSI domain) in its extracellular region. Here, we describe Plxdc2 expression in the embryonic mouse, with particular emphasis on the developing central nervous system. Using light microscopy and optical projection tomography (OPT), we analyse RNA in situ hybridization patterns and expression of two reporter genes, $\beta$-geo (a fusion of $\beta$-galactosidase to neomycin phosphotransferase) and placental alkaline phosphatase (PLAP) in a Plxdc2 gene trap mouse line (KST37; [Leighton, P.A., Mitchell, K.J., Goodrich, L.V., Lu, X., Pinson, K., Scherz, P., Skarnes, W.C., Tessier-Lavigne, M., 2001. Defining brain wiring patterns and mechanisms through gene trapping in mice. Nature 410, 174-179]). At mid-embryonic stages (E9.5-E11.5) Plxdc2- $\beta$ geo expression is prominent in a number of patterning centres of the brain, including the cortical hem, midbrain-hindbrain boundary and the midbrain floorplate. Plxdc2 is expressed in other tissues, most notably the limbs, lung buds and developing heart, as well as the spinal cord and dorsal root ganglia. At E15.5, expression is apparent in a large number of discrete nuclei and structures throughout the brain, including the glial wedge and derivatives of the cortical hem. Plxdc2Bgeo expression is particularly strong in the developing Purkinje cell layer, especially in the posterior half of the cerebellum. The PLAP marker is expressed in a number of axonal tracts, including the posterior commissure, mammillotegmental tract and cerebellar peduncle. We compare Plxdc2-ßgeo expression in the embryonic brain with the much more restricted expression of the related gene Plxdcl and with members of the Wnt family (Wnt3a, Wnt5a and Wnt8b) that show a striking overlap with Plxdc2 expression in certain areas.
\end{abstract}

(c) 2007 Elsevier B.V. All rights reserved.

Keywords: Plxdc2; Plxdc1; TEM7R; TEM7; KST37; Wnt1; Wnt3a; Wnt5a; Wnt8b; PLAP secretory trap; OPT, optical projection tomography; Cortical hem; Cajal-Retzius cells; Midbrain-hindbrain boundary; Glial wedge; Purkinje cell layer; AER, apical ectodermal ridge; Mammillotegmental tract; Posterior commissure; Cerebellar peduncle

\section{Results and discussion}

During neurodevelopment, patterning of the neuropeithelium and establishment of cell fates is followed by (or sometimes linked with) migration of cells to appropriate positions and guidance of growing axons along stereotyped pathways to appropriate targets. A number of conserved

\footnotetext{
Corresponding author. Tel.: +3531896 3067; fax: +35316798558.

E-mail address: Kevin.Mitchell@tcd.ie (K.J. Mitchell).
}

families of molecules involved in axon guidance have been identified in several species. These include families of guidance cues such as the netrins, semaphorins, slits, and ephrins (Dickson, 2002; Yu and Bargmann, 2001). In addition, a number of classically defined morphogens, including Wnts, BMPs, and Shh have also recently been found to act in axon guidance (reviewed in Charron and Tessier-Lavigne, 2005). These molecules and their receptors are characterised by a variety of extracellular motifs involved in cell-cell interactions, dimerisation or autoregulation. In 
addition to these well-characterised proteins, a large number of novel transmembrane protein-encoding genes containing such motifs are predicted in mammalian genomes, many of which are expressed in the developing brain (unpublished data) and are good candidates to be involved in axon guidance or other aspects of nervous system development.

The PLAP secretory trap method was designed and developed to screen for novel axon guidance receptors in the mouse (Leighton et al., 2001). Previous studies utilising this method isolated a gene trap insertion in a novel gene (mouse line KST37; Leighton et al., 2001), since named plexin domain-containing 2 (official MGI gene symbol $P l x d c 2$ ). This gene encodes a type I transmembrane protein of 530 amino acids, characterised by an extracellular region of weak nidogen homology and a plexin repeat or PSI domain, a domain found in known axon guidance molecules such as semaphorins and plexins as well as integrins and a small number of other molecules (Bork et al., 1999). The PSI domain has been shown to be involved in autoregulatory intramolecular contacts in a number of cases (Bunch et al., 2006; Mould et al., 2005; Zang and Springer, 2001). A related gene (now called Plxdcl) was isolated in a screen for genes upregulated in human tumour endothelium and named tumour endothelial marker 7 (TEM7) (Carson-Walter et al., 2001; St Croix et al., 2000). Plxdc2 is thus also referred to as TEM7-related (TEM7R). The parallels between angiogenesis and nerve growth have been noted before and several molecules originally identified in axon guidance have been directly implicated in angiogenesis (Basile et al., 2006; Carmeliet and Tessier-Lavigne, 2005).

In this study, we investigate $P l x d c 2$ expression in the mouse at a number of stages of embryonic development, paying particular attention to its expression in the developing nervous system. We compare this with the much more restricted expression of $P l x d c 1$ in the embryonic brain and with a number of Wnt genes that show a striking overlap with $P l x d c 2$ expression in certain areas.

\subsection{Characterisation of a PLAP secretory trap insertion in Plxdc2}

The PLAP secretory trap method was designed to identify axon guidance genes in the mouse by simultaneous mutation of the trapped gene and transgenic labelling of neurons that normally express it and their axons. The endogenous promoter of the trapped gene drives production of a bicistronic transcript that encodes two proteins, a fusion of the endogenous protein to $\beta$-geo (a fusion of $\beta$-gal to neomycin phosphotransferase) and placental alkaline phosphatase (PLAP), which is translated independently using an internal ribosome entry site. It is important to note that both markers report gene expression and not necessarily endogenous protein localisation; PLAP labels axons of neurons expressing the trapped gene, whereas the $\beta$-geo fusion protein remains membrane bound inside cell bodies. Comparisons of corresponding $\mathrm{X}$-gal and PLAP stainings confirm that PLAP reliably labels the axons of neurons stained with X-gal (Leighton et al., 2001). Studies of previously trapped genes have shown that both the PLAP and $\beta$ gal markers faithfully reflect reported expression patterns (Leighton et al., 2001), although perdurance of the $\beta$-geo fusion protein may be an issue in comparing the dynamics of expression to results from in situ hybridisation techniques.

Using a candidate primer approach, we characterised the exact site of the PLAP secretory trap insertion in the KST37 mouse line to intron 3-4 of the Plxdc2 gene (Ensembl:ENSMUST00000028081; Supplemental figure 1).

\subsection{Plxdc-Bgeo expression in the embryo}

Expression of Plxdc2- $\beta$ geo in the embryo was investigated by whole mount X-gal staining of heterozygous gene trap mice at embryonic day (E)9.5, E11.5 and in the brain of E15.5 embryos. At E9.5, Plxdc2- $\beta$ geo expression was observed in the floor of the midbrain and in an anterior and medial area of the telencephalon that includes the choroid plexus epithelium and presumptive cortical hem (Fig. 1). Expression of Plxdc2- $\beta$ geo was also evident in the midbrain-hindbrain boundary (MHB), a major organising
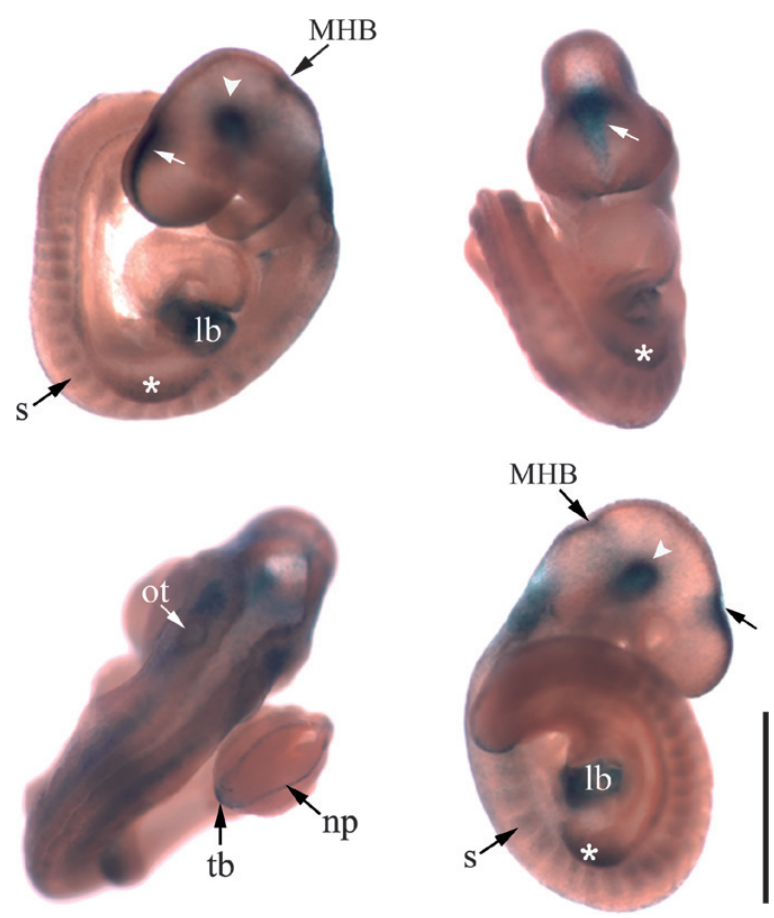

Fig. 1. Plxdc2- $\beta$ geo expression in the E9.5 mouse. X-gal staining of heterozygous gene trap mice illustrated cellular Plxdc2- $\beta$ geo expression in discrete regions of the developing embryo. Arrow, anteromedial region of the telencephalon that includes the choroid plexus epithelium and presumptive cortical hem; arrowhead, floor of midbrain; asterisk, limb-bud; lb, lung bud; MHB, midbrain-hindbrain boundary; ot, otic vesicle; np, lip of neuropore; tb, tail bud; s, Plxdc2- $\beta$ geo expression within the somites. Scale bar $1.25 \mathrm{~mm}$. 
centre of the developing brain (Raible and Brand, 2004). Within the trunk region, Plxdc2- $\beta$ geo expression occurred in a segmented pattern within the somites. At later stages of development, Plxdc2- $\beta$ geo expression is found in the dorsal root ganglia (DRG, see below) and expression at E9.5 is thus most likely to include the neural crest-derived spinal primordia which give rise to the sensory cells of the DRG. Plxdc2- $\beta$ geo expression was also evident in the limb buds, the lung buds and in the otic vesicle (Fig. 1). In the most caudal region of the embryo, expression is seen in the lateral edges of the neuroepithelium in the region of the still open posterior neuropore (future dorsal midline) and in the extreme distal tip of the tail bud (Fig. 1).

By E11.5, Plxdc2- $\beta$ geo expression was more complex and widespread, and its intricate expression pattern became increasingly difficult to document using classical microscopy and photography techniques. We therefore utilised Optical Projection Tomography (OPT), a method for 3D optical microscopy, to visualise Plxdc2-ßgeo expression in the E11.5 mouse (Fig. 2). Rather than scanning through different depths of focus in the specimen to reconstruct the $3 \mathrm{D}$ object (as in confocal microscopy), OPT maximises the depth of focus, capturing projected optical density images of the whole specimen (Sharpe et al., 2002). Computer software then reconstructs the original $3 \mathrm{D}$ information from 400 images taken through one complete 360 degree revolution. The $3 \mathrm{D}$ computer object can then be viewed as a series of virtual sections in any orientation or can be viewed from "external" view-points (when volume rendered). Fig. 2 shows both external views (b-e and g) and virtual sections (f, h, i, and j).

OPT highlighted Plxdc2- $\beta$ geo expression in restricted domains in a number of areas of the E11.5 embryo (Fig. 2; Supplementary data, video 1) including the brain, limb bud, sensory ganglia, branchial arches, heart, lung buds (Figs. 2 and 3 ) and urogenital tract (data not shown). In the eye, cellular Plxdc2- $\beta$ geo expression was restricted to a region of localised expression in the centre of the lens (Fig. 2a and b). In both hind and fore limb buds, Plxdc2- $\beta$ geo was expressed in broad domains of distal mesenchyme, extending more proximally on the posterior side (Fig. 2a and d) with a reduced level at the extreme distal tip (Fig. 2f and h). Expression is also seen in the apical ectodermal ridge, a major signalling centre involved in the proximal-distal patterning of the developing vertebrate limb (Fig. $2 \mathrm{f}$ and inset in $\mathrm{h}$ ). Continued Plxdc2- $\beta$ geo expression was also evident in regions of the developing otic vesicle (Fig. 2b). Plxdc2-ßgeo expression within the trunk at E11.5 had resolved to a clear restriction within the developing DRG (Fig. 2f). This pattern of expression agrees with previously published data at E12.5, which showed Plxdc2- $\beta$ geo expression in the DRG and PLAP expression in the corresponding axons (Leighton et al., 2001). These data also showed reporter gene expression in motor and sympathetic preganglionic axons
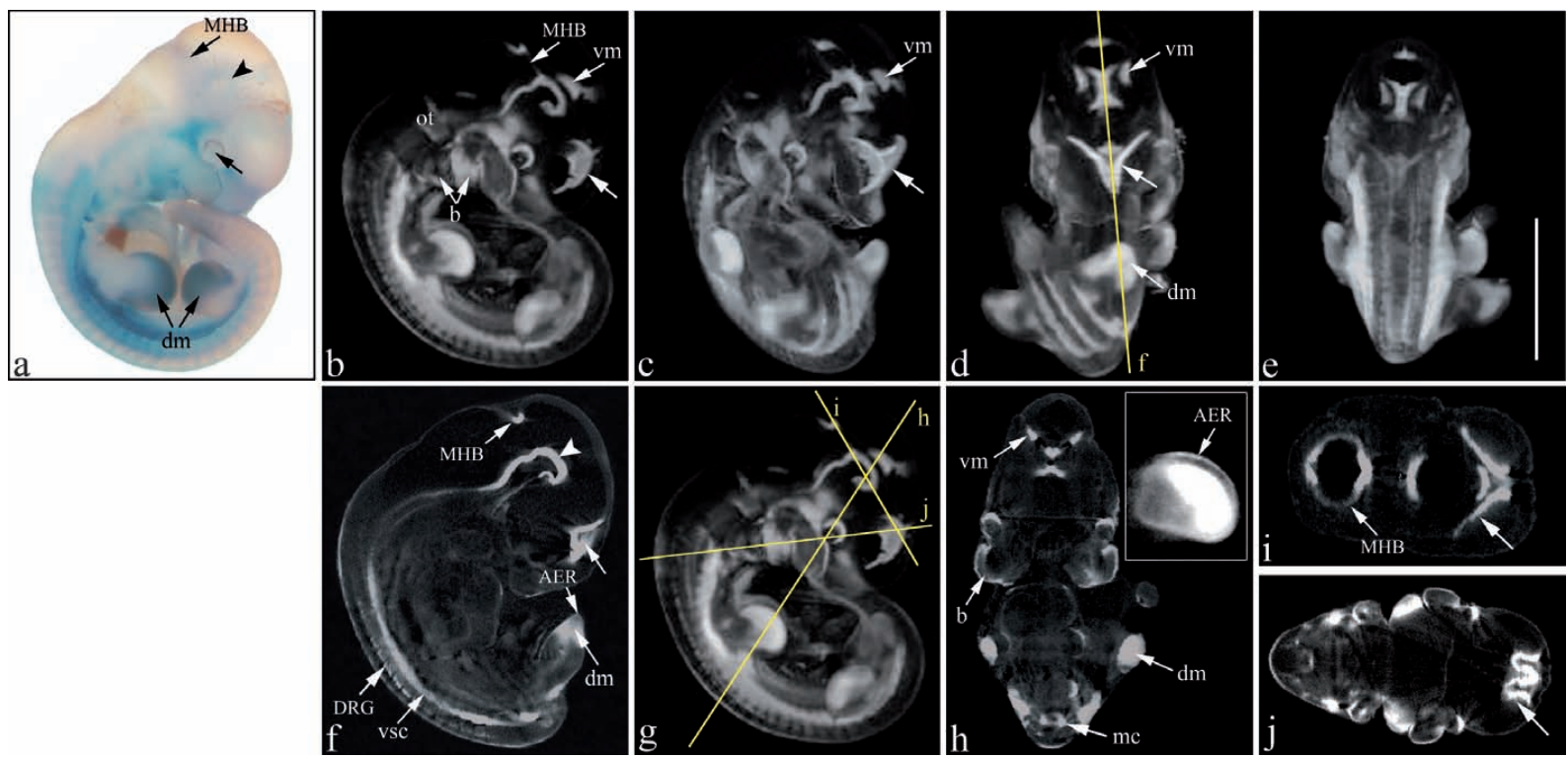

Fig. 2. Plxdc2-ßgeo expression in the E11.5 mouse revealed by OPT analysis of X-gal stained heterozygous $P l x d c 2$ gene trap embryos. a, whole mount image of an X-gal stained E11.5 embryo. b-e and g, still images from different external viewing angles of a volume rendered specimen (Supplemental data, movie 1). $\mathrm{f}$ and $\mathrm{h}-\mathrm{j}$, virtual sections through the specimen. Insert in $\mathrm{h}$, sagittal section through the forelimb showing Plxdc2- $\beta$ geo expression in the AER. Panels $\mathrm{i}$ and $\mathrm{j}$, anterior to the right. Apparent optic cup staining is owing to pigmentation of this area in C57Bl6 mice. a: arrow, Plxdc2- $\beta$ geo expression in the lens of the eye. b-j; arrow, cortical hem and developing choroid plexus. Arrowhead, floor of midbrain; AER, apical ectodermal ridge; b, Plxdc2- $\beta$ geo expression within the branchial arches; $\mathrm{dm}$, Plxdc2- $\beta$ geo expression in the distal mesenchyme of the limb; DRG, dorsal root ganglia; mc, motor column; MHB, midbrain-hindbrain boundary; ot, otic vesicle; vm, clusters of Plxdc2- $\beta$ geo expression in the ventral midbrain; vsc, ventral spinal cord, including floorplate and motor column. Scale bar: a-g and j, $2.9 \mathrm{~mm} ; \mathrm{h}, 2.6 \mathrm{~mm} ; \mathrm{i}, 2 \mathrm{~mm}$. 
and the floorplate of the spinal cord (Leighton et al., 2001). In the present study, expression is seen in the ventral spinal cord, including the floorplate from an anterior limit at the midbrain, and the motor column of the neural tube from an anterior limit at the level of prevertebrae $\mathrm{C} 2 / \mathrm{C} 3$ (Fig. 2f and h). A broadband of expression is also seen in the lateral plate mesoderm.

In the E11.5 brain, Plxdc2-ßgeo expression was observed in a number of discrete areas, including important patterning centres. The broad expression seen in the medial telencephalon at E9.5 has evolved to a clearly defined domain (Fig. $2 \mathrm{i}$ and j). By this stage the telencephalic vesicles have expanded and are separated by a medial invagination of the dorsal midline due to low levels of proliferation combined with high levels of apoptosis (Furuta et al., 1997). Plxdc2$\beta$ geo expression was observed throughout the medial invagination encompassing the cortical hem and choroid plexus epithelium $(\mathrm{CPe})$ and extending slightly caudal in the medial diencephalon (Fig. $2 \mathrm{i}$ and j, arrow). The cortical hem is crucial for normal cortical development and disruption of the area can lead to severe hippocampal abnormalities (Shimogori et al., 2004). At E11.5, a complete ring of Plxdc2-Bgeo expression was evident at the MHB (Fig. 2i) with significantly more intense expression both dorsally and ventrally. Elsewhere in the brain, Plxdc2- $\beta$ gal continues to be expressed in the floor of the midbrain although this expression now extends into the hindbrain. Two clusters of cellular Plxdc2- $\beta$ geo expression were also evident in the ventral midbrain, lateral to and distinct from the floor plate expression (Fig. 2b, c, d, and h).

The specificity of the reporter gene expression was confirmed by whole-mount in situ hybridisation with a $P l x d c 2$ antisense riboprobe (Fig. 3). At E11.5, in situ hybridisation showed the same overall pattern of expression as the Plxdc2- $\beta$ geo reporter gene, although there was a reduction in the relative intensity in certain areas, particularly in the cortical hem and the limbs (Fig. 3a, d, and e). These areas gave a stronger in situ hybridisation signal at E10.5 (Fig. 3b and c). This modest disparity could be due to technical differences in the protocols used for in situ hybridisation on E10.5 and E11.5 embryos (the former being freshly prepared and the latter having been frozen), or possibly to perdurance of the Plxdc2- $\beta$ geo fusion protein. Despite this difference, in situ hybridisation confirmed Plxdc2 expression in the cortical hem, the MHB, the floor of the midbrain and in all of the areas highlighted by X-gal staining of heterozygous gene trap embryos. Later expression in specific brain areas was also confirmed by in situ hybridisation
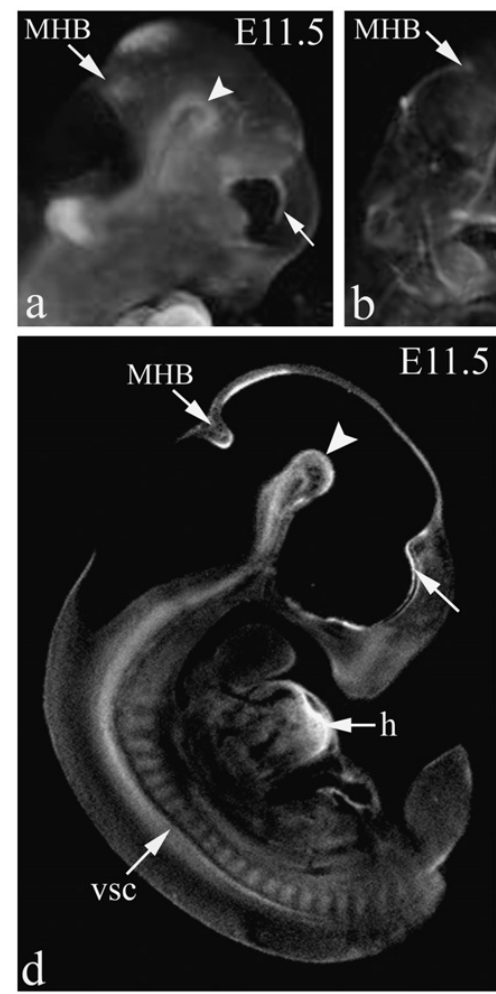
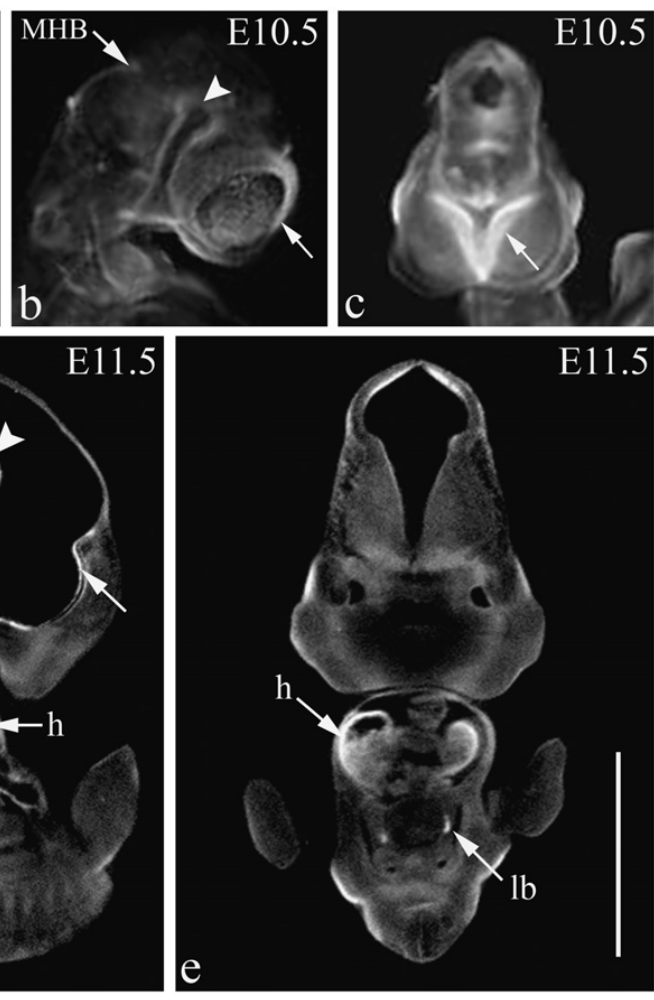

Fig. 3. In situ hybridisation confirms the Plxdc2- $\beta$ geo expression pattern. Whole-mount Plxdc2 in situ hybridisation on E10.5 (b,c) and E11.5 (a,d,e) wildtype embryos analysed by OPT. a-c, still images from different external viewing angles of volume rendered specimens. $\mathrm{d}$ and e, virtual sections through an E11.5 embryo showing reduced Plxdc2 expression in the cortical hem and MHB. Arrow, cortical hem and choroid plexus; arrowhead, floor of midbrain; h, heart; lb, lung bud; MHB, midbrain-hindbrain boundary; vsc, ventral spinal cord, including floorplate and motor column. Scale bar: a, d and e 2.7 mm; b and c $1.9 \mathrm{~mm}$. 
(data not shown). A control sense probe for Plxdc2 showed a low level non-specific background staining (data not shown).

\subsection{Comparative definition of Plxdc- $\beta$ geo expression within the cortical hem and $M H B$}

We noted remarkable similarities between the expression of Plxdc2- $\beta$ geo and that of a number of members of the Wnt family, several of which are expressed in the cortical hem and MHB, for example. Wnt1 is essential for the development of the posterior midbrain and anterior hindbrain and is expressed in a thin ring at the MHB as early as E9.5 (Panhuysen et al., 2004). Wnt3a expression can be used to distinguish the structure of the cortical hem as early as E10.5 (Grove et al., 1998). By E11.5, Wnt5a and Wnt8b are also expressed in the cortical hem (Grove et al., 1998; Shimogori et al., 2004; Tole et al., 2000).

In order to more closely define the domains of Plxdc2$\beta$ geo expression within brain structures, we compared its expression at E11.5 to that of Wnt1, Wnt $3 a$, Wnt5a, and $W n t 8 b$, examined and viewed with the same OPT methodology (Fig. 4). Comparison with Wntl and Wnt5a confirms co-expression with Plxdc2- $\beta$ geo at the MHB and in the

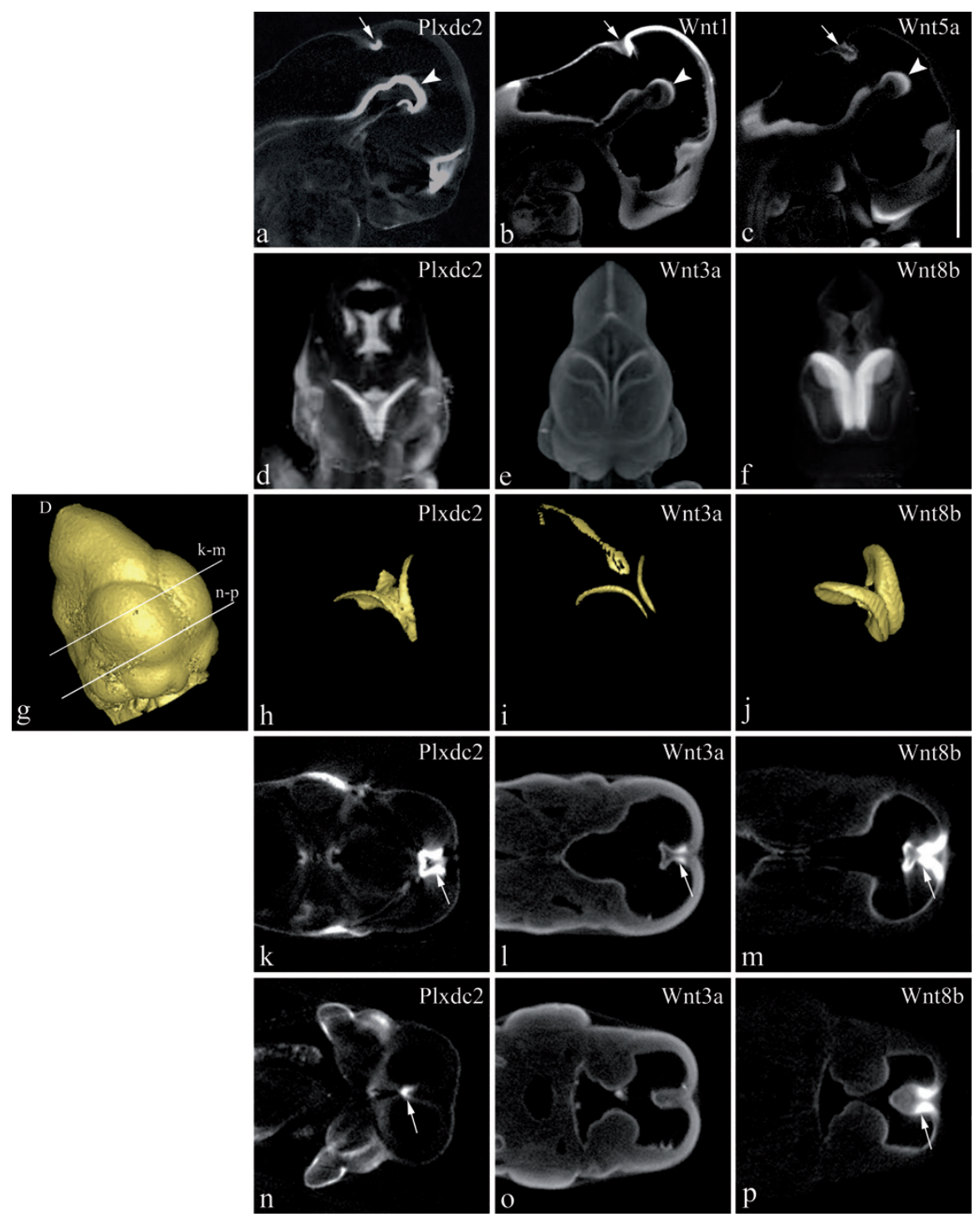

Fig. 4. Definition of Plxdc2- $\beta$ geo expression within the cortical hem and MHB at E11.5 by comparison to Wnt1, Wnt3a, Wnt5a and Wnt8b. a-c and k-p, virtual sections and $\mathrm{d}-\mathrm{f}$, frontal external views of heads of volume rendered specimens. $\mathrm{g}-\mathrm{j}$, surface rendered views of whole head (g) and stained region of anteromedial telencephalon $(\mathrm{h}-\mathrm{j})$. Lines in $\mathrm{g}$ indicate plane of sections in $\mathrm{k}-\mathrm{m}$ and $\mathrm{n}-\mathrm{p}$. a-c: arrow, MHB; arrowhead, floor of midbrain. $\mathrm{k}-\mathrm{m}$ : arrow indicates position of cortical hem, the extent of which is shown by Wnt3a expression in 1. n-p: arrows indicate differential staining at rostral levels, as described in the text. Scale bar: $\mathrm{a}-\mathrm{j}, 1.45 \mathrm{~mm} ; \mathrm{k}-\mathrm{p}, 2.2 \mathrm{~mm}$. 
floor of the midbrain (Fig. 4a, b, and c), but unlike Wnt1, Plxdc2- $\beta$ geo is not detected in the dorsal midline of the midbrain. In the floorplate, Plxdc2- $\beta$ geo expression is more extensive rostrocaudally than either of the Wnt genes and is not restricted to the ventricular aspect. Wnt5a expression also shows striking similarities with that of Plxdc2 in the limb (Yamaguchi et al., 1999) and in the glial wedge (see below and Keeble et al., 2006).

The extent of the expression domain in the medial cerebral cortex was more closely defined by comparison with domains of Wnt3a, Wnt $8 b$ (Fig. 4d-p) and Wnt5a expression (data not shown). Fig. 4d-f show dorsal external views of the midbrain-forebrain regions of $3 \mathrm{D}$ reconstructed (volume rendered) specimens. Fig. 4h-j represents the overall shape and size of the domains by showing them in isolation at a constant viewing angle, whereas Fig. 4k-p shows comparable virtual sections. As previously described (e.g., Grove et al., 1998), Wnt3a is expressed precisely within the cortical hem (Fig. 4i and l) as well as the dorsal midline of the more caudal brain. Wnt $8 b$ is expressed in a broader domain encompassing the cortical hem but extending also into the choroid plexus medially, and more laterally along the walls of the telencephalic vesicles including the region of the prospective hippocampus (Fig. $4 \mathrm{j}$ and $\mathrm{m}$ ). The domain of Plxdc2- $\beta$ geo expression is less broad than Wnt8b but also includes the choroid plexus and the prospective hippocampus (Fig. 4h and k). Rostrocaudally, the midline expression of Plxdc2- $\beta$ geo is more extensive than either Wnt gene; as mentioned earlier, it extends caudally into the dorsal midline of the diencephalon and Fig. $4 \mathrm{n}-\mathrm{p}$ shows that rostrally, Plxdc2-ßgeo is expressed at a level where $W n t 3 a$ is no longer expressed and $W n t 8 b$ is localised more laterally.

\subsection{Plxdc2-Bgeo expression in the E15.5 brain}

Expression of Plxdc2- $\beta$ geo in the E15.5 brain was investigated by X-gal staining of $100 \mu \mathrm{m}$ sections from heterozygous $P l x d c 2$ gene trap mice (Fig. 5). An intricate pattern of Plxdc2- $\beta$ geo expression was observed in a number of nuclei throughout the brain as well as in the neuroepithelium. In the forebrain, regions of cellular Plxdc2- $\beta$ geo expression include the glial wedge and the developing medial septum (Fig. 5a-c). Expression is evident in the choroid plexus (derived from the $\mathrm{CPe}$ ) and derivatives of the cortical hem, namely the fimbria and the dentate gyrus of the developing hippocampus as well as Cajal-Retzius cells in the marginal zone of the developing cortex (Fig. 5d-f). Plxdc2- $\beta$ geo expression is also observed in discrete nuclei within the hypothalamus, habenula, pretectum and amygdala (Fig. $5 \mathrm{f}-\mathrm{k}$ ). In more caudal regions, Plxdc2- $\beta$ geo is also expressed within the tegmentum and pons in the midbrain reticular formation and pontine reticular formation (Fig. 5n-s). Plxdc2- $\beta$ geo expression was notable at the border region of the superior colliculus and the pons (Fig. 5q) and within the principal sensory trigeminal nucleus and trigeminal motor nucleus (Fig. $5 \mathrm{r}$ and s). In the hindbrain, extensive Plxdc2- $\beta$ geo expression was evident in the floorplate and in distinct compartments of the developing Purkinje cell layer of the cerebellum as well as many nuclei of the medulla oblongata, including the vestibular nuclei (Fig. 5t-x). (Expression in the developing Purkinje cell layer has also been described at P0 (Leighton et al., 2001)).

Staining in several areas including the glial wedge and fimbria, for example, indicates that Plxdc2- $\beta$ geo expression is, at least in part, glial in nature. Neuronal Plxdc2 expression was also confirmed by PLAP staining of several axon tracts in the E15.5 brain (Fig. 6). PLAP staining was evident in axons of the posterior commissure which crosses the midline at the dorsal midbrain, connecting right and left pretectal areas (Fig. 6a-d). PLAP expression also occurred in axons of the mammillotegmental tract which project from the mammillary nuclei to the tegmentum (Fig. 6c-d). In the hindbrain, strong PLAP staining was evident in the cerebellar peduncle connecting the cerebellum and the medulla oblongata (Fig. 6e-g). The sagittal view also highlights the uneven expression in the developing cerebellum with much greater expression in the caudal half at this midsagittal level (Fig. 6b and d). Very discrete expression at the border between the inferior colliculus and developing cerebellum is also evident in this view (Fig. $6 \mathrm{~b}$ and d). Widespread vascular Plxdc2 expression in the brain is also apparent by PLAP staining (Fig. 6).

\subsection{Plxdc2-PLAP expression in the eye and ear at E15.5}

PLAP staining of $100 \mu \mathrm{m}$ sections through the head illustrated continued $P l x d c 2$ expression in the eye and ear at E15.5 (Fig. 7). PLAP staining within the eye was restricted to the proliferating region of the inner neuroblastic layer and the cuboidal epithelium of the lens (Fig. 7a). Sagittal sections through the ear illustrated $P l x d c 2$ expression in the cochlea. PLAP staining was evident in the ventral epithelium and a sharp border existed between ventral and dorsal sides (Fig. 7b and c). Neuronal Plxdc2 expression was also illustrated by axonal staining within the trigeminal ganglion (Fig. 7c).

\subsection{Plxdc1 expression}

Plxdc1/TEM7 shows significant homology to Plxdc2, sharing the same domain architecture and exhibiting 53\% amino acid identity between the two proteins in the mouse. An investigation of $P l x d c l$ expression in the brain was facilitated by the creation of a Plxdcl "targeted trap" mouse line (Friedel et al., 2005). In this case, the PLAP secretory trap vector was fused to gene-specific homology arms, thus utilizing homologous recombination to insert the vector into the intron between exons 11 and 12 of the $P l x d c 1$ gene.

Expression of Plxdc1- $\beta$ geo in the E16.5 brain was investigated by X-gal staining of $100 \mu \mathrm{m}$ sections from homozygous Plxdcl targeted trap mice (Fig. 8); (in this case homozygous mutants were examined as they give 


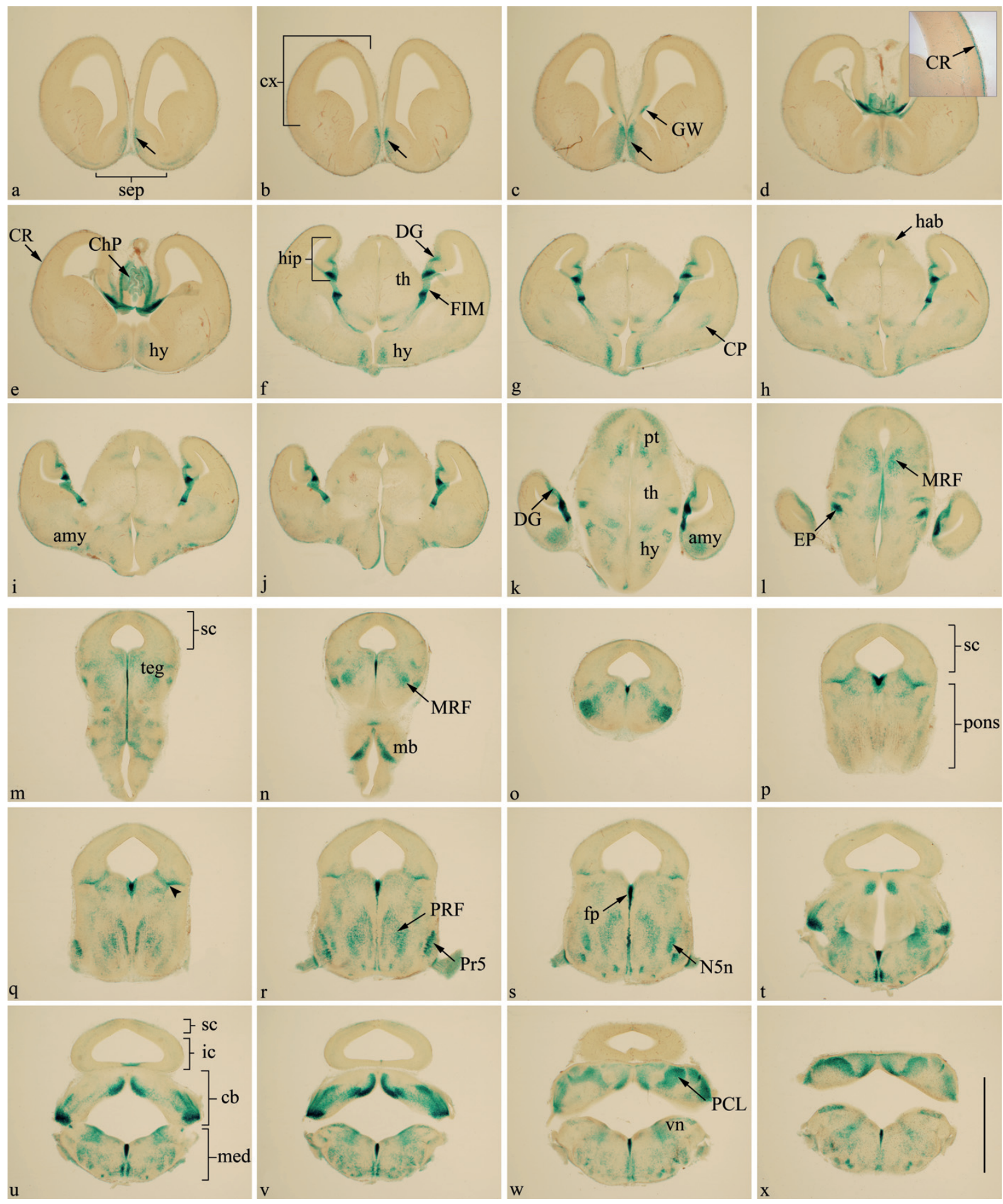

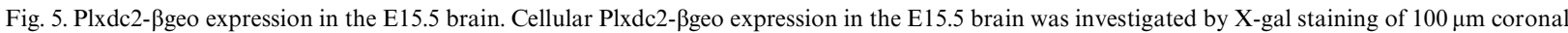

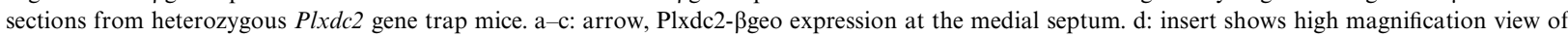
presumptive Cajal-Retzius cells. q: arrowhead, clusters of Plxdc2-ßgeo expression at the border region of the tectum and the pons. amy, amygdala; cb, cerebellum; ChP, choroid plexus; CP, caudate putamen; CR, Cajal-Retzius cells; cx, cortex; DG, dentate gyrus; EP, entopeduncular nucleus; FIM, fimbria; fp, floorplate; GW, glial wedge; hab, habenula; hip, hippocampus; hy, hypothalamus; ic, inferior colliculus; mb, mammillary bodies; med, medulla oblongata; MRF, midbrain reticular formation; N5n, trigeminal motor nucleus; PCL, Purkinje cell layer; PRF, pontine reticular formation; Pr5, principle sensory trigeminal nucleus; pt, pretectum; sc, superior colliculus; sep, septum; teg, tegmentum; th, thalamus; vn, vestibular nuclei. Scale bar $1.5 \mathrm{~mm}$. 


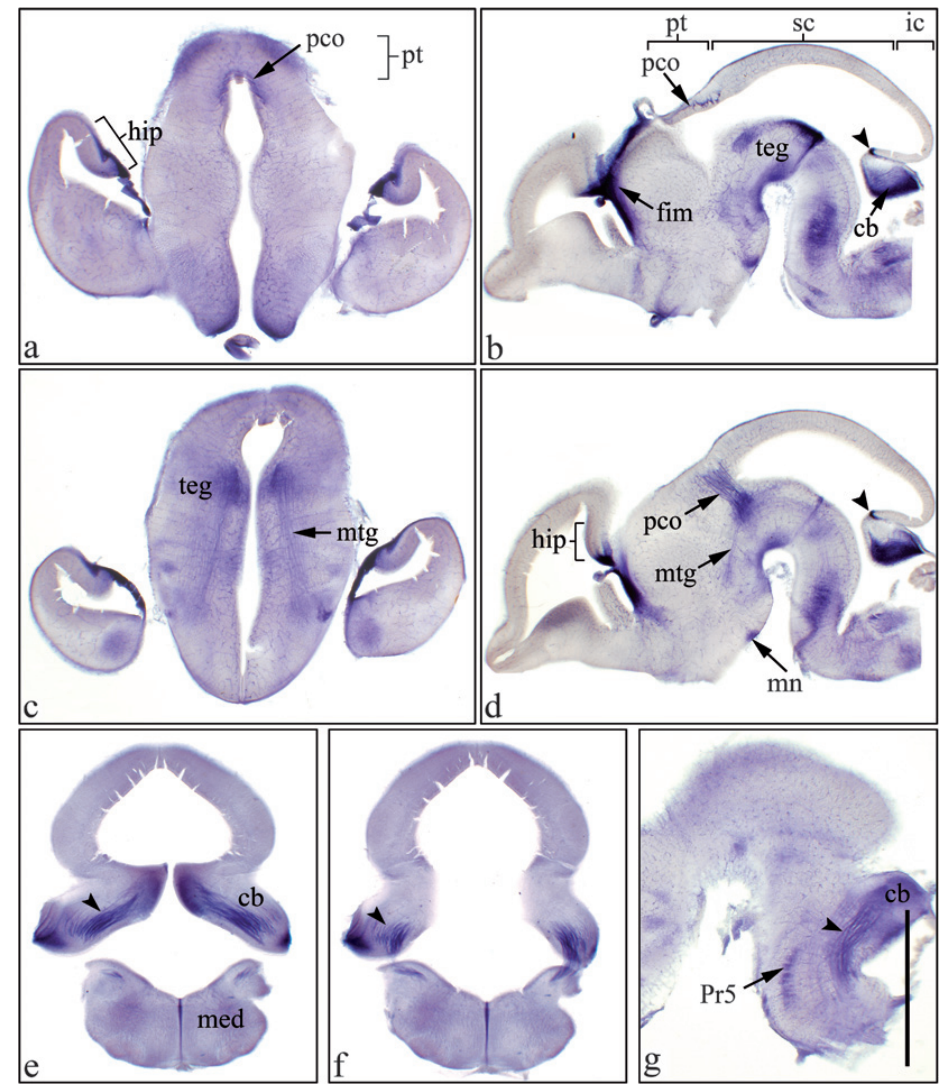

Fig. 6. Neuronal Plxdc2 (PLAP) expression in the E15.5 brain. a and c, coronal sections through the E15.5 brain illustrating PLAP staining in axons of the posterior commissure and the mammillotegmental tract. $\mathrm{b}$ and $\mathrm{d}$, corresponding sagittal sections illustrating the trajectory of these fibre tracts. $\mathrm{e}$ and $\mathrm{f}$, coronal sections showing PLAP staining in axons of the cerebellar peduncle projecting between the cerebellum and the medulla oblongata. g, corresponding sagittal section illustrating the trajectory of this tract. $\mathrm{b}$ and $\mathrm{d}$ : arrowhead, border of inferior colliculus and cerebellum. e-g: arrowhead, cerebellar peduncle. cb, cerebellum; fim, fimbria; hip, hippocampus; ic, inferior colliculus; mn, mammillary nucleus; mtg, mammillotegmental tract; pco, posterior commissure; Pr5, principal sensory trigeminal nucleus; pt, pretectum; sc, superior colliculus; teg, tegmentum. Scale bar: a and c $1.5 \mathrm{~mm} ; \mathrm{b}$ and d-f $1.75 \mathrm{~mm} ; \mathrm{g} 1.3 \mathrm{~mm}$.
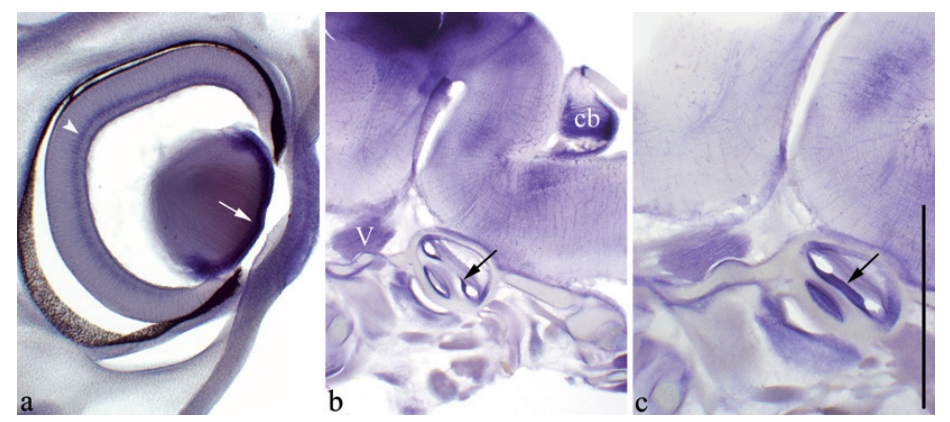

Fig. 7. Plxdc2 (PLAP) expression in the eye and ear at E15.5. a, coronal section through the eye showing PLAP expression. arrowhead, proliferating region of the inner neuroblastic layer; arrow, cuboidal epithelium of the lens. b and c, sagittal sections through the head illustrating PLAP expression in the ventral epithelium of the cochlea (arrows) and in axons of the trigeminal ganglion (V). cb, cerebellum. Scale bar: a, $0.7 \mathrm{~mm} ; \mathrm{b}, 2 \mathrm{~mm} ; \mathrm{c}, 1.5 \mathrm{~mm}$.

more intense staining but appear phenotypically normal). Plxdc1-ßgeo expression in the brain differed greatly to that of Plxdc2- $\beta$ geo. At E16.5, Plxdc1- $\beta$ geo expression was restricted to a single paired midbrain nucleus lying ventral to the tectum (Fig. 8a), in a location consistent with the pedunculopontine tegmental nucleus. Neuroepi- thelial expression was also evident at the fourth ventricle (Fig. 8b). More widespread expression of Plxdc1- $\beta$ geo was observed in neonatal and postnatal brain sections (data not shown), in accord with in situ hybridisation and immunohistochemistry results in the rat (Lee et al., 2005, 2006). Specific expression in the Purkinje cells and para- 


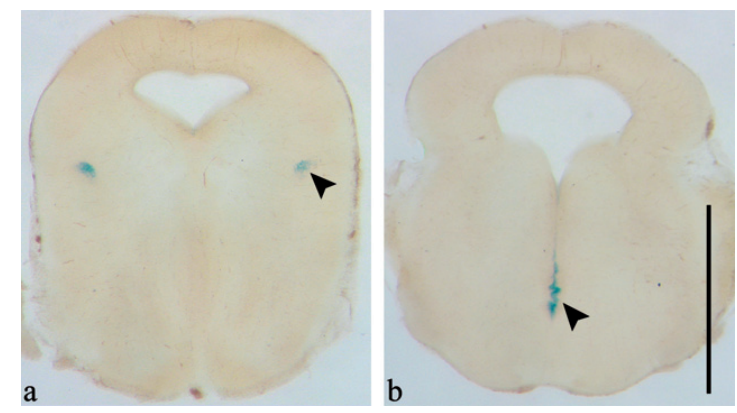

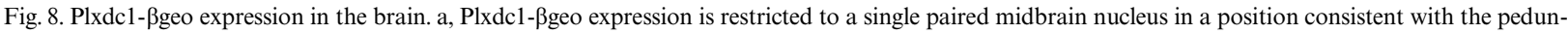
culopontine tegmental nucleus (arrowhead). b, neuroepithelial Plxdc1- $\beta$ geo expression is evident at the fourth ventricle (arrowhead). Scale bar $1.5 \mathrm{~mm}$.

ventricular nucleus of the hypothalamus at these later stages, for example, (data not shown) matches observations in the rat precisely (Lee et al., 2005), confirming the specificity and sensitivity of the reporter genes. At E10.5, whole mount X-gal staining of homozygous gene trap mice illustrated no Plxdc1-ßgeo expression.

\section{Experimental procedures}

\subsection{Characterisation of a PLAP secretory trap insertion in Plxdc2}

A candidate primer approach was employed in order to characterise the site of vector insertion. A series of forward primers was designed from the wildtype $P l x d c 2$ genomic sequence at $1.5 \mathrm{~kb}$ intervals and reverse primers were designed to the vector sequence. Genomic DNA from heterozygous Plxdc2 mice was used as template for the PCR reaction. A $2.2 \mathrm{~kb}$ band was amplified using one primer combination and sequenced in order to isolate the exact site of insertion.

\subsection{Genotyping of mice}

Genotyping of gene trap mice was carried out by PCR. Forward and reverse primers were designed to wildtype genomic sequence close to the site of vector insertion. An additional reverse primer was designed to the vector sequence in order to amplify mutated fragments. Genomic DNA was extracted from small tail sections by digestion in Boston Buffer $(50 \mathrm{mM}$ Tris $\mathrm{pH} 8.0,50 \mathrm{mM} \mathrm{KCl}, 2.5 \mathrm{mM}$ EDTA, $0.45 \% \mathrm{NP} 40,0.45 \%$ Tween 20, Proteinase K) overnight at $56^{\circ} \mathrm{C}$ and used as template for the PCR. The primer sequences were as follows: Plxdc2; forward primer to wildtype sequence 5'-GAGAGCTACTTAGGCTGATTCTGT-3', reverse primer to wildtype sequence $5^{\prime}$-CCTCGACAGTAATTCAACCT GCTG-3', reverse primer to vector sequence $5^{\prime}$-GCTACCGGCTAA AACTTGAGACCT- $3^{\prime}$. Plxdc1; forward primer to wildtype sequence $5^{\prime}$ TCTCTCTACATAGCTCTGGCTGTC-3', reverse primer to wildtype sequence $5^{\prime}$-AGTCAGGTCTGAGGTCAGCTGTCT-3', reverse primer to vector sequence $5^{\prime}$-ACTTCCGGAGCGGATCTCAAACTC-3'.

\subsection{Visualisation of reporter genes}

Whole embryos were fixed in $0.2 \%$ glutaraldehyde $(0.1 \mathrm{M}$ phosphate buffer, $2 \mathrm{mM} \mathrm{MgCl}_{2}, 5 \mathrm{mM}$ EGTA) for $30 \mathrm{~min}$. Following fixation, three washes were carried out at room temperature in washing buffer $(0.1 \mathrm{M}$ phosphate buffer, $2 \mathrm{mM} \mathrm{MgCl}, 0.02 \% \mathrm{NP} 40,0.1 \%$ sodium deoxycholate). Embryos were transferred to X-gal staining solution $\left(1 \mathrm{mg} \mathrm{ml}^{-1}\right.$ $\mathrm{X}$-gal, $5 \mathrm{mM} \mathrm{K}_{4} \mathrm{Fe}(\mathrm{CN})_{6}, 5 \mathrm{mM} \mathrm{K}{ }_{3} \mathrm{Fe}(\mathrm{CN})_{6}$ in embryo washing buffer $)$ and incubated at $37{ }^{\circ} \mathrm{C}$ until colour developed. Stained embryos were post-fixed in $4 \%$ paraformaldehyde (PFA)/phosphate buffered saline (PBS) at $4{ }^{\circ} \mathrm{C}$ overnight in order to preserve staining patterns and stored in PBS.
Brains for X-gal staining were fixed in $1 \%$ glutaraldehyde for $5 \mathrm{~h}$. Sections $(100 \mu \mathrm{m})$ were collected using a vibrating microtome (Leica, model VT1000S) and free floating sections were stained for $\beta$ gal activity (as previously described). Following staining, sections were post-fixed in $4 \%$ PFA/PBS, air dried onto glass slides and mounted in Aqua Polymount (Polysciences Inc.).

E15 brains and whole heads for PLAP staining were fixed overnight in $4 \%$ PFA/PBS at $4{ }^{\circ} \mathrm{C}$. Fixed tissues were embedded in $6 \%$ agar and $100 \mu \mathrm{m}$ sections collected using a vibrating microtome. Sections were air dried onto glass slides. Following incubation in PBS for $45 \mathrm{~min}$ at $65^{\circ} \mathrm{C}$ to inactivate endogenous phosphatases, PLAP activity was detected with AP staining buffer $\left(0.1 \mathrm{mg} \mathrm{ml}^{-1}\right.$ 5-bromo-4-chloro-3-indolyl phosphate, $1 \mathrm{mg} \mathrm{ml}^{-1}$ nitroblue tetrazolium in $100 \mathrm{mM}$ Tris-HCL pH $9.5,100 \mathrm{mM}$ $\mathrm{NaCl}, 5 \mathrm{mM} \mathrm{MgCl}$ ). Sections were dehydrated in increasing concentrations of methanol and cleared in benzyl benzoate: benzylalcohol (BB:BA, 2:1). Following rehydration, cleared sections were mounted in Aqua Polymount.

\subsection{In situ hybridisation}

In situ hybridisation was carried out largely according to Wilkinson (1992) with minor adjustments. Hybridisation times were extended to 2-3 days at $65^{\circ} \mathrm{C}$. Final post-hybridisation washes were in $0.2 \% \mathrm{SSC} /$ $0.1 \% \mathrm{CHAPS}$, three times for $20 \mathrm{~min}$ at $65^{\circ} \mathrm{C}$. Antisense probes used are represented by the following Genbank sequences: Plxdc2; nucleotides 1524-2188 on NM_026162. Wnt1; nucleotides $138-2345$ on BC005449. Wnt3a; nucleotides 2310-2676 on NM_009522. Wnt5a; nucleotides 193 2324 on BC018425. Wnt8b; nucleotides $942-1634$ on NM_011720. Sense control probes were routinely employed in each experiment. Mice strains: E10.5, C57BL/6JOlaHsd (Harlan); E11.5, CD1.

\subsection{Optical projection tomography}

In situ hybridised or X-gal stained embryos were rinsed briefly in water, and embedded in $1 \%$ low melting point agarose. Once set, the block of agarose was cut in the desired orientation and affixed to a metal mount. Specimens were then dehydrated overnight in $100 \%$ methanol and cleared in BB:BA (2:1). OPT was carried out as described (Sharpe et al., 2002) on a prototype scanner built at the MRC Human Genetics Unit Edinburgh (now commercially available from BioOptonics). Software for 3D reconstruction, volume rendering and analysis was kindly provided by James Sharpe and the Edinburgh Mouse Atlas Project (MRC Human Genetics Unit).

\section{Acknowledgements}

We thank James Sharpe and the Edinburgh Mouse Atlas Project (MRC Human Genetics Unit) for provision and support of the OPT system. This work was supported 
by Science Foundation Ireland grants to K.J.M (01/F.1/ B006) and to P.M (02/IN1/B267).

\section{Appendix A. Supplementary data}

Supplementary data associated with this article can be found, in the online version, at doi:10.1016/j.modgep.2006.12.002.

\section{References}

Basile, J.R., Castilho, R.M., Williams, V.P., Gutkind, J.S., 2006. Semaphorin 4D provides a link between axon guidance processes and tumor-induced angiogenesis. Proc. Natl. Acad. Sci. USA 103, 9017 9022.

Bork, P., Doerks, T., Springer, T.A., Snel, B., 1999. Domains in plexins: links to integrins and transcription factors. Trends Biochem. Sci. 24, 261-263.

Bunch, T.A., Helsten, T.L., Kendall, T.L., Shirahatti, N., Mahadevan, D., Shattil, S.J., Brower, D.L., 2006. Amino acid changes in Drosophila alphaPS2betaPS integrins that affect ligand affinity. J. Biol. Chem. 281, 5050-5057.

Carmeliet, P., Tessier-Lavigne, M., 2005. Common mechanisms of nerve and blood vessel wiring. Nature 436, 193-200.

Carson-Walter, E.B., Watkins, D.N., Nanda, A., Vogelstein, B., Kinzler, K.W., St Croix, B., 2001. Cell surface tumor endothelial markers are conserved in mice and humans. Cancer Res. 61, 6649-6655.

Charron, F., Tessier-Lavigne, M., 2005. Novel brain wiring functions for classical morphogens: a role as graded positional cues in axon guidance. Development 132, 2251-2262.

Dickson, B.J., 2002. Molecular mechanisms of axon guidance. Science 298, 1959-1964.

Friedel, R.H., Plump, A., Lu, X., Spilker, K., Jolicoeur, C., Wong, K., Venkatesh, T.R., Yaron, A., Hynes, M., Chen, B., Okada, A., McConnell, S.K., Rayburn, H., Tessier-Lavigne, M., 2005. Gene targeting using a promoterless gene trap vector ("targeted trapping") is an efficient method to mutate a large fraction of genes. Proc. Natl. Acad. Sci. USA $102,13188-13193$.

Furuta, Y., Piston, D.W., Hogan, B.L., 1997. Bone morphogenetic proteins (BMPs) as regulators of dorsal forebrain development. Development 124, 2203-2212.

Grove, E.A., Tole, S., Limon, J., Yip, L., Ragsdale, C.W., 1998. The hem of the embryonic cerebral cortex is defined by the expression of multiple Wnt genes and is compromised in Gli3-deficient mice. Development $125,2315-2325$.
Keeble, T.R., Halford, M.M., Seaman, C., Kee, N., Macheda, M., Anderson, R.B., Stacker, S.A., Cooper, H.M., 2006. The Wnt receptor Ryk is required for Wnt5a-mediated axon guidance on the contralateral side of the corpus callosum. J. Neurosci. 26, 5840-5848.

Lee, H.K., Bae, H.R., Park, H.K., Seo, I.A., Lee, E.Y., Suh, D.J., Park, H.T., 2005. Cloning, characterization and neuronal expression profiles of tumor endothelial marker 7 in the rat brain. Brain Res. Mol. Brain Res. 136, 189-198.

Lee, H.K., Kang, D.S., Seo, I.A., Choi, E.J., Park, H.T., Park, J.I., 2006. Expression of tumor endothelial marker $7 \mathrm{mRNA}$ and protein in the dorsal root ganglion neurons of the rat. Neurosci. Lett. 402, 71-75.

Leighton, P.A., Mitchell, K.J., Goodrich, L.V., Lu, X., Pinson, K., Scherz, P., Skarnes, W.C., Tessier-Lavigne, M., 2001. Defining brain wiring patterns and mechanisms through gene trapping in mice. Nature 410, 174-179.

Mould, A.P., Travis, M.A., Barton, S.J., Hamilton, J.A., Askari, J.A., Craig, S.E., Macdonald, P.R., Kammerer, R.A., Buckley, P.A., Humphries, M.J., 2005. Evidence that monoclonal antibodies directed against the integrin beta subunit plexin/semaphorin/integrin domain stimulate function by inducing receptor extension. J. Biol. Chem. 280, 4238-4246.

Panhuysen, M., Vogt Weisenhorn, D.M., Blanquet, V., Brodski, C., Heinzmann, U., Beisker, W., Wurst, W., 2004. Effects of Wnt1 signaling on proliferation in the developing mid-/hindbrain region. Mol. Cell Neurosci. 26, 101-111.

Raible, F., Brand, M., 2004. Divide et Impera-the midbrain-hindbrain boundary and its organizer. Trends Neurosci. 27, 727-734.

Sharpe, J., Ahlgren, U., Perry, P., Hill, B., Ross, A., Hecksher-Sorensen, J., Baldock, R., Davidson, D., 2002. Optical projection tomography as a tool for 3D microscopy and gene expression studies. Science 296, 541-545.

Shimogori, T., Banuchi, V., Ng, H.Y., Strauss, J.B., Grove, E.A., 2004. Embryonic signaling centers expressing BMP, WNT and FGF proteins interact to pattern the cerebral cortex. Development 131, 5639-5647.

St Croix, B., Rago, C., Velculescu, V., Traverso, G., Romans, K.E., Montgomery, E., Lal, A., Riggins, G.J., Lengauer, C., Vogelstein, B., Kinzler, K.W., 2000. Genes expressed in human tumor endothelium. Science 289, 1197-1202.

Tole, S., Ragsdale, C.W., Grove, E.A., 2000. Dorsoventral patterning of the telencephalon is disrupted in the mouse mutant extra-toes $(J)$. Dev. Biol. 217, 254-265.

Wilkinson, D.G., 1992. In situ hybridisation: A practical approach. IRL Press, Oxford.

Yamaguchi, T.P., Bradley, A., McMahon, A.P., Jones, S., 1999. A Wnt5a pathway underlies outgrowth of multiple structures in the vertebrate embryo. Development 126 (6), 1211-1223.

Yu, T.W., Bargmann, C.I., 2001. Dynamic regulation of axon guidance. Nat. Neurosci. 4 (Suppl), 1169-1176.

Zang, Q., Springer, T.A., 2001. Amino acid residues in the PSI domain and cysteine-rich repeats of the integrin beta2 subunit that restrain activation of the integrin alpha(X)beta(2). J. Biol. Chem. 276, 6922-6929. 